\title{
STABILITY RESULTS OF SOME ABSTRACT EVOLUTION EQUATIONS
}

\begin{abstract}
N.S. HOANG
Abstract. The stability of the solution to the equation $\dot{u}=A(t) u+G(t, u)+f(t), t \geqslant 0, u(0)=$ $u_{0}$ is studied. Here $A(t)$ is a linear operator in a Hilbert space $H$ and $G(t, u)$ is a nonlinear operator in $H$ for any fixed $t \geqslant 0$. We assume that $\|G(t, u)\| \leqslant \alpha(t)\|u\|^{p}, p>1$, and the spectrum of $A(t)$ lies in the half-plane $\operatorname{Re} \lambda \leqslant \gamma(t)$ where $\gamma(t)$ can take positive and negative values. We proved that the equilibrium solution $u(t) \equiv 0$ to the equation is Lyapunov stable under persistantly acting perturbations $f(t)$ if $\sup _{t \geqslant 0} \int_{0}^{t} \gamma(\xi) d \xi<\infty$ and $\int_{0}^{\infty} \alpha(\xi) d \xi<\infty$. In addition, if $\int_{0}^{t} \gamma(\xi) d \xi \rightarrow-\infty$ as $t \rightarrow \infty$, then we proved that the equilibrium solution $u(t) \equiv 0$ is asymptotically stable under persistantly acting perturbations $f(t)$. Sufficient conditions for the solution $u(t)$ to be bounded and for $\lim _{t \rightarrow \infty} u(t)=0$ are proposed and justified.
\end{abstract}

Mathematics subject classification (2010): 34G20, 37L05, 44J05, 47J35.

Keywords and phrases: evolution equations, stability, Lyapunov stable, asymptotically stable.

\section{REFERENCES}

[1] L. Cesari, Asymptotic Behavior and Stability Problems in Ordinary Differential Equations, Springer-Verlag, Berlin, 1963.

[2] E. Coddington And N. Levinson, Theory of Ordinary Differential Equations, McGrawHill, NewYork, 1955.

[3] Y. Daleckit And M. KReIn, Stability of solutions of differential equations in Banach spaces, Amer. Math. Soc., Providence, RI, 1974.

[4] B. Demidovich, Lectures on Mathematical Theory of Stability, Nauka: Moscow, 1967. (in Russian)

[5] P. Hartman, Ordinary Differential Equations, Wiley, NewYork, 1964.

[6] A. LyApunOv, Collected Works, II. Acad. Sci., Moscow, 1954. (in Russian)

[7] A. G. Ramm And N. S. HoAng, Dynamical Systems Method and Applications, Theoretical Developments and Numerical examples, Wiley, Hoboken, 2012.

[8] A. G. Ramm, Asymptotic stability of solutions to abstract differential equations, J. Abstr. Differ. Equ. Appl., 1 (2010), N1, 27-34.

[9] A. G. Ramm, Stability of solutions to some evolution problems, Chaotic Modeling and Simulation (CMSIM), 1 (2011), 17-27.

[10] A. G. Ramm, A stability result for abstract evolution problems, Math. Meth. Appl. Sci., 36 (2012), N4, 422-426.

[11] A. G. RAmM, Stability of the solutions to evolution problems, Mathematics, 1 (2013), 46-64.

[12] A. G. RAmm, Stability of solutions to abstract evolution equations with delay, J. Math. Anal. Appl., 396 (2012), 523-527.

[13] R. Temam, Infinite-dimensional Dynamical Systems in Mechanics and Physics, Springer-Verlag, NewYork, 1997. 